\title{
Improved methodology for performing the inverse Abel transform of flame images for colour ratio pyrometry
}

\author{
Jochen A.H. Dreyer ${ }^{1,2}$, Radomir I. Slavchov ${ }^{1,3}$, Eric J. Rees ${ }^{1}$, Jethro Akroyd ${ }^{1,2}$, Maurin

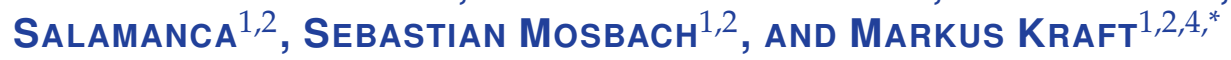 \\ ${ }^{1}$ Department of Chemical Engineering and Biotechnology, University of Cambridge, Philippa Fawcett Drive, Cambridge, CB3 OAS, United Kingdom \\ ${ }^{2}$ Cambridge Centre for Advanced Research and Education in Singapore (CARES), CREATE Tower, 1 Create Way, Singapore 138602 \\ ${ }^{3}$ School of Engineering and Materials Science, Queen Mary University of London, Mile End Road, London, E1 4NS, United Kingdom \\ ${ }^{4}$ School of Chemical and Biomedical Engineering, Nanyang Technological University, 62 Nanyang Drive, Singapore 637459 \\ *Corresponding author: mk306@cam.ac.uk
}

Compiled February 4, 2019

A new method is presented for performing the Abel inversion by fitting the line-of-sight projection of a predefined intensity distribution (FLiPPID) to the recorded 2D projections. The aim is to develop a methodology that is less prone to experimental noise when analysing the projection of antisymmetric objects, in this case co-flow diffusion flame images for colour ratio pyrometry. A regression model is chosen for the light emission intensity distribution of the flame cross-section as a function of the radial distance from the flame centre-line. The forward Abel transform of this model function is fitted to the projected light intensity recorded by a colour camera. For each of the three colour channels, the model function requires three fitting parameters to match the radial intensity profile at each height above the burner. This results in a very smooth Abel inversion with no artifacts such as oscillations or negative values of the light source intensity, as is commonly observed for alternative Abel inversion techniques, such as the basis-set expansion (BASEX) or onion-peeling. The advantages of the new FLiPPID method are illustrated by calculating the soot temperature and volume fraction profiles inside a co-flow diffusion flame, both being significantly smoother than those produced by the alternative inversion methods. The developed FLiPPID methodology can be applied to numerous other optical techniques for which smooth inverse Abel transforms are required. ๑ 2019 Optical Society of America

http://dx.doi.org/10.1364/ao.XX.XXXXXX

\section{INTRODUCTION}

Laminar flames are widely used for fundamental studies of soot formation [1-4] and the synthesis of materials [5-8]. Characterising such systems with accurate and reliable techniques is vital for understanding the processes controlling particle formation in flames and is an ongoing field of research [9]. The employed techniques can generally be divided into intrusive and nonintrusive methods. Examples for the former are measurements of the flame temperature with a thermocouple, soot sampling to measure particle size distributions $[1,10]$, or thermophoretic soot collection for ex situ analysis [11, 12]. Whenever possible, non-intrusive techniques are preferred to avoid perturbation of the system. One such technique that has gained increasing attention in recent years, is colour ratio pyrometry [9, 13-15]. Here, the colour and intensity of the visible light emitted by hot soot are used to infer their temperature and volume fraction [16, 17]. No expensive equipment is required, making pyrometry an economic and rapid method to obtain $2 \mathrm{D}$ soot temperature and volume fraction data.

One of the main challenges in colour ratio pyrometry is the reconstruction of the flame cross-section emission profile, $R(r, z)$, from the projected area profile $P(x, z)$ recorded by a camera (Fig. $1)$. In case of optically thin flames (i.e., negligible soot selfabsorption [9]) with axial symmetry, the recorded 2D projection $P(x, z)$ and the 3D flame emission density $R(r, z)$ are linked through the forward and reverse Abel transforms [18-21]:

$$
\begin{gathered}
P(x, z)=2 \int_{x}^{\infty} \frac{R(r, z) r}{\sqrt{r^{2}-x^{2}}} \mathrm{~d} r \\
R(r, z)=-\frac{1}{\pi} \int_{r}^{\infty} \frac{\partial P(x, z)}{\partial x} \frac{1}{\sqrt{x^{2}-r^{2}}} \mathrm{~d} x,
\end{gathered}
$$

where $z$ is the height above the burner (HAB), $r$ is the cylindrical coordinate and $x$ is the projected coordinate (distance from the central axis). 


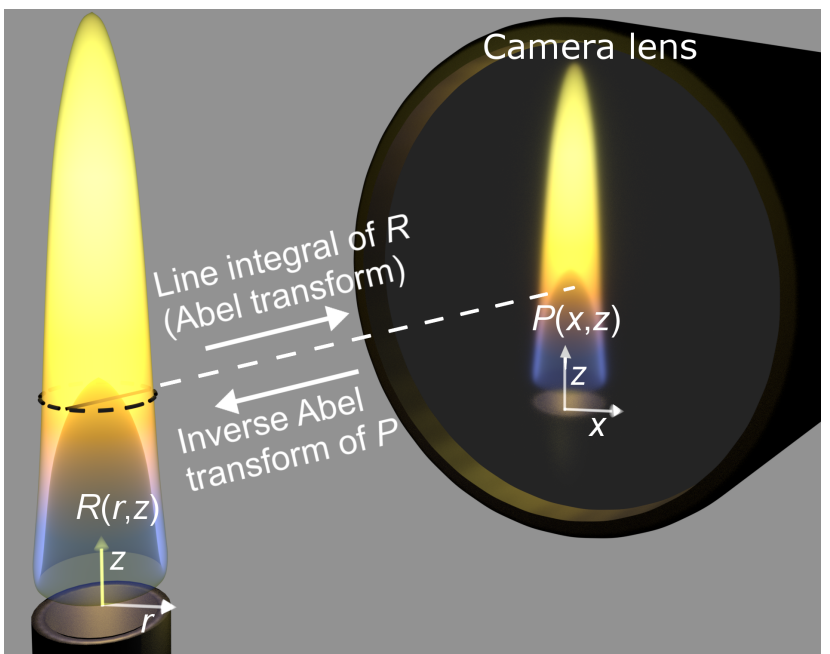

Fig. 1. Illustration of the relationship between the original light emission distribution $R(r, z)$ of the flame cross-section and its projection $P(x, z)$.

Unfortunately, applying the inverse transform directly to experimental data recorded for $P$ is not feasible because the experimental noise is significantly amplified in the reconstructed density, especially close to the axis of symmetry [22]. The reason is that the recorded signal is not sensitive enough to the emission intensity near the centre. Numerous methods have been developed to circumvent this information deficit and to reduce the noise amplification upon image reconstruction (reviewed by Buie et al. [23]). Examples are integral transform techniques such as the Fourier-Hankel method [24] and its extension to filter noise [25], iterative methods [26], or fitting of polynomials or splines to the observed data [27-29]. Two techniques especially common in flame pyrometry are the basis-set expansion (BASEX) $[18,19]$ and the onion-peeling method combined with a Tikhonov regularisation [20,21]. These two approaches are similar in that they regularise the reconstruction by supplying the measured data with additional information - a condition for a predefined level of spatial smoothness (set by smoothing and filtering parameters). Whilst these two methods are computationally cheap, they are often unable to dampen the noise amplification in $R(r, z)$ towards the axis of symmetry despite the regularisation. The noisy image reconstruction has a significant effect on the soot temperatures $T$ and volume fractions $f_{\mathrm{v}}$ computed from $R$. This is especially problematic if flame centre-line values are the desired quantity. For example, estimating sooting propensities of fuels with the yield sooting indices (YSIs) requires the maximum of $f_{\mathrm{V}}$, which is often in the flame centre $[13,30,31]$.

The purpose of this work is to describe a new Abel inversion technique that is less sensitive to noise and produces smooth intensity cross-section reconstructions from their 2D projections. The proposed method is based on fitting the line-of-sight projection of a predefined intensity distribution (FLiPPID) to the recorded projection. The predefined intensity distribution $R(r)$ is tailored to the geometry of the studied signal source, applying physically motivated constraints such as a non-negative light intensity $(R>0)$. The function of choice is specific to the application, but the methodology is general and can be applied to any steady, optically thin, axisymmetric system.

\section{EXPERIMENTS}

The system studied here was a co-flow diffusion flame. The analysed signal originated from hot soot particles emitting black body radiation (Fig. 1). The flame was stabilised using a Yale burner [32] fed with $7 \mathrm{~g} / \mathrm{h}$ pre-vaporised (Bronkhorst CEM) $n$ heptane in $200 \mathrm{~mL} / \mathrm{min}$ argon carrier gas. The fuel/carrier gas mixture was delivered through heated lines to a central 1/4" stainless steel tube (inner diameter $0.218 ")$. A $50 \mathrm{~L} / \mathrm{min}$ co-flow of air was passed through a 3 " honeycomb mesh (0.017" wire diameter, $18 \times 18$ mesh). The flame images were recorded with a Blackfly S (BFS-U3-32S4C-C, FLIR Integrated Imaging Solutions, Inc.) having a $1 / 1.8$ " Sony IMX252 CMOS sensor with 2048x1536 pixels (pixel size $3.45 \mu \mathrm{m}$ ). The camera lens was a MVL25M23 from Thorlabs with an aperture set to $f / 1.4$ and a focal length of $25 \mathrm{~mm}$. The distance between the camera and the flame was $25 \mathrm{~cm}$, resulting in a pixel width corresponding to $1 / 34 \mathrm{~mm}$ in the focal plane through the centre of the flame. A BG-7 filter (Thorlabs) was used to balance the intensity ratios of the three colour channels and to block infra-red light. The assumption of parallel ray acquisition (orthographic imaging) was taken as valid given the small flame radius $(<4 \mathrm{~mm})$ compared to the object distance $(25 \mathrm{~cm})$. A more comprehensive discussion on the effect of non-parallel ray collection on potential errors in the Abel conversion is given by Walsh et al. [33]. The image processing was performed on single frames to avoid blurring of the flame edges upon averaging. A flow chart of the image processing algorithm is shown in Fig. 2 while a more detailed description of the individual steps is given in Appendix A. Links to the archived and current versions of the corresponding Python code can be found at the end of Appendix B in Ref. [1] and [2], respectively.

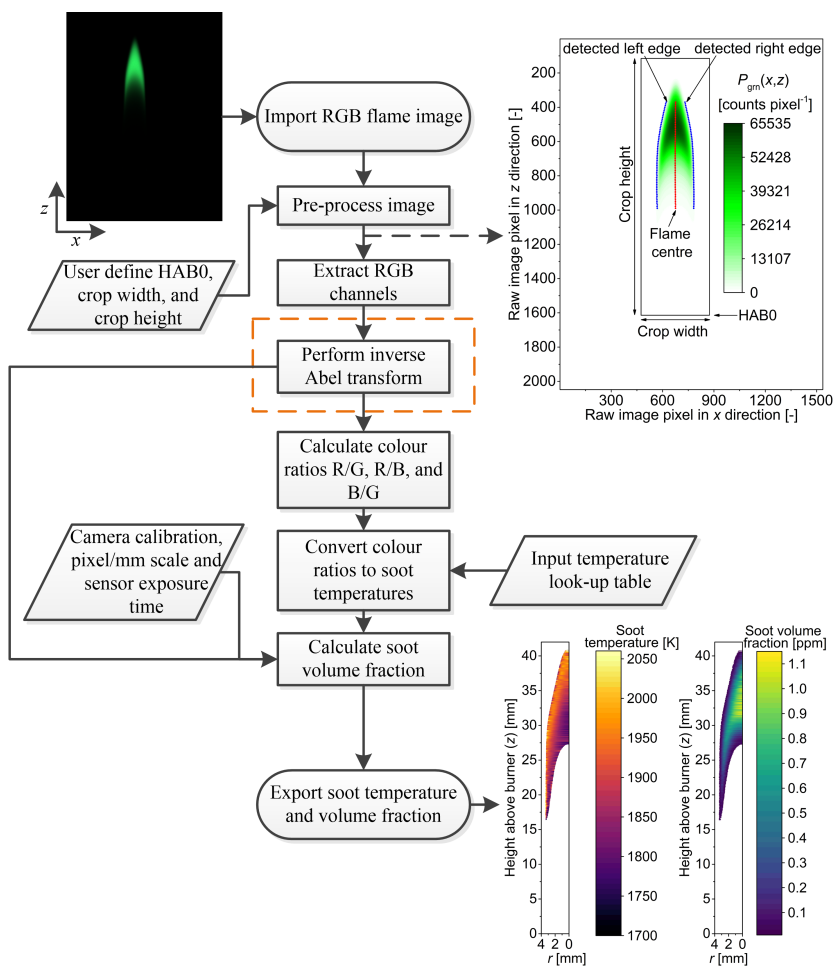

Fig. 2. Flow chart of the algorithm for converting the recorded RGB flame image to cross-sectional profiles of the soot temperature and volume fraction. 


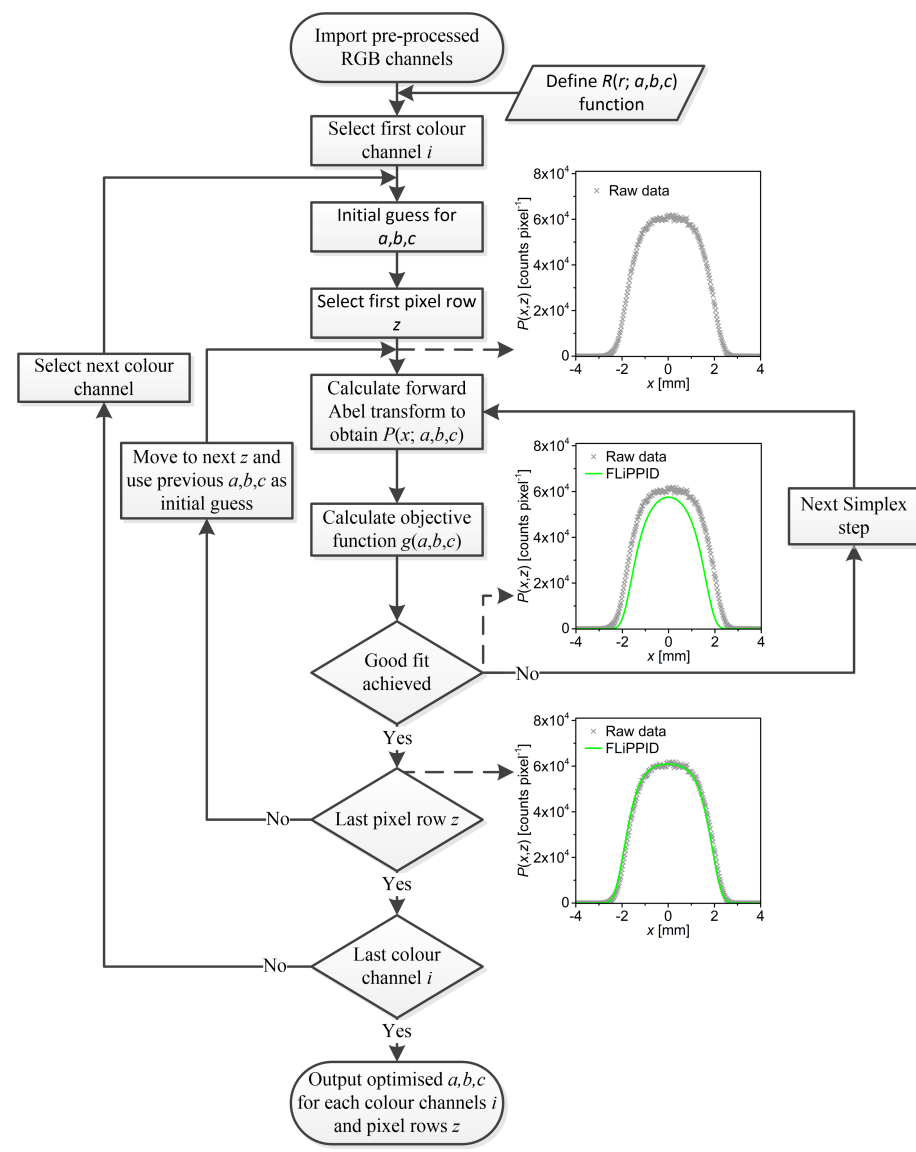

Fig. 3. Flow chart for the application of the FLiPPID method using the example of RGB flame images.

\section{FLIPPID METHODOLOGY}

The FLiPPID method developed here requires the definition of a suitable function $R(r ; a, b, c \ldots), a, b, c \ldots$ being fitting parameters, that is able to describe the intensity distribution at the crosssection of the studied signal source. The forward Abel transform (Eq. 1) of $R$ was computed numerically to obtain $P(x ; a, b, c \ldots)$. A residual sum-of-squares objective function $g(z ; a, b, c, \ldots)$ measuring the difference between the calculated projection $P$ and the corresponding experimentally recorded $2 \mathrm{D}$ projection was minimised. A Simplex optimisation was used here but other methods can be selected in the provided Python code (Ref. [1] and [2] in Appendix B).

The fitting procedure was repeated for all pixel rows in $z$ and thus all height above the burner (HAB), and for all three colour channels. A flow chart of the FLiPPID methodology is shown in Fig. 3. A detailed descriptions of each step can be found in Appendix B.

By first intent, the FLiPPID method imposes reasonable constraints on the model function $R(r ; a, b, c .$.$) . The first condition$ $R$ has to fulfill is non-negativity - light intensity has to be positive at all $r$. The second constraint is simplicity - among all possible functions that fulfill a predefined criterion for agreement with the data, the reconstruction with the fewest parameters is selected as most likely. Additional non-restrictive "convenience" conditions were used to help with the selection of a family of model functions to test:

(i) The test functions should be applicable at all $z$. (ii) The test functions should be able to represent the known shape of the source density. In this case, functions with a maximum intensity either located on the centre-line or located in the wings of the flame. This shape of $R$ has been well-documented with methods that do not rely on inverse Abel transforms, e.g., laser-induced incandescence [17, 32].

(iii) While testing different model functions, it was noted that $R$ should decay as $\exp \left(-k r^{n}\right)$ where $n \geq 2$ to achieve reasonable fits to the observed data.

The functions considered as possible models for $R$ were: (i) e to the power of polynomials (exp-polynomials); (ii) polynomials multiplied by exp-polynomials; (iii) various derivatives of $1 / \cosh (r)$; (iv) sums of these, and modifications of these with terms proportional to $r^{1 / 2}, \ln r$ and $1 /(r+a)$ added to the polynomials.

The agreement between a test function $R$ and the data was considered sufficiently good when the optimal value of the objective function $g$ was by no more than $0.5 \%$ different from the intrinsic residual sum-of-squares of the experimental data, $g_{\text {int }}$, tested at $10-15$ different $z$. The latter parameter $g_{\text {int }}$ was defined as the minimal value of the sum-of-squares for the recorded data at a given $z$ and a set of regression models for $P$ generated by fitting polymonials of increasing order $n$ to the data. The optimal sum-of-squares of the polynomial models decreased with $n$ until a well-defined plateau value - namely, $g_{\text {int }}$ - was reached at $n=15-30$. The optimal sum-of-squares for the polynomial models does not decrease further until $n$ approaches the number of data points, producing oscillating polynomial functions following the noise of the measured data.

The plateau value $g_{\text {int }}$ is a practically model-independent characteristic of the recorded data, and is used as a benchmark for how well a model can possibly fit the data. The permissible difference between $g$ and $g$ int $(0.5 \%)$ was chosen to be smaller by a factor of 5-10 than the typical reproducibility of the recorded signal (discussed below), to ensure that no statistically significant information is lost by imposing the simplicity condition on $R$.

\section{RESULTS}

The least-parametric function $R(r ; a, b, c)$ that fulfills the imposed conditions is:

$$
R(r)=\frac{a}{b \sqrt{\pi}} \exp \left[c\left(\frac{r}{b}\right)^{2}-\left(\frac{r}{b}\right)^{6}\right], \quad a, b \in \mathbb{R}^{+}, \quad c \in \mathbb{R} .
$$

Here, $a$ is characteristic of the amplitude of $R, b$ is of the order of magnitude as the radius of the flame, and $c$ defines the radial location of the maximum. No other positive 4-, 5- or 6-parameter test function for $R$ was found that led to a $g$ significantly closer to $g_{\text {int }}$ than the one given by Eq. 3 .

This function was further used for the analysis of all recorded images and cross-sections. The computation of the Abel transform of Eq. 3 as required for the fitting procedure is the computationally most demanding step of FLiPPID. However, the integrals can easily be tabulated into a look-up table by writing Eqs. 1 and 3 in the equivalent form:

$$
P(x)=\frac{2 a}{\sqrt{\pi}} I(x / b, c),
$$

where $I$ is the integral 


$$
I(X, c)=\int_{0}^{\infty} \exp \left[c\left(\sigma^{2}+X^{2}\right)-\left(\sigma^{2}+X^{2}\right)^{3}\right] \mathrm{d} \sigma .
$$

The computation of this look-up table and the optimisation to find the best fit from it is included in the provided Python code (Ref. [1] and [2] in Appendix B).
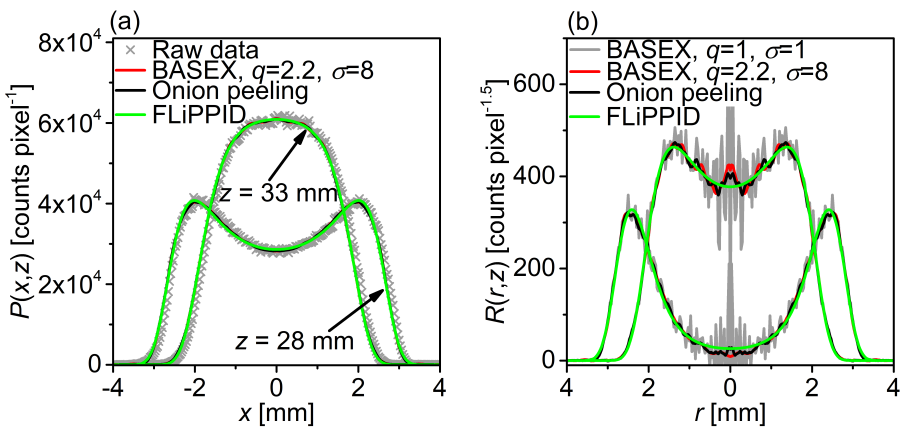

Fig. 4. (a) Recorded green light projection of the flame at $z=$ 33 and $28 \mathrm{~mm}$ (highlighted in Fig. 5a) together with FLiPPID and smoothed/filtered $P(x)$ using BASEX and onion peeling. (b) Reconstructed cross-section density from the data in (a) using the three different methods as well as BASEX without smoothing/filtration $(q=1, \sigma=1)$.

Fig. 4a compares flame projections at two HAB (recorded by the green channel) with the corresponding fits with Eq. 3 and its forward Abel transform (Eq. 1). Excellent agreement between the fitted and recorded data was obtained despite the simplicity of the regression model and the diverse profile shapes at different $\mathrm{HAB}$. Fits at other $z$ and for other colour channels led to equally good or better results (the projection for $z=28$ $\mathrm{mm}$ led to the worst fit, with standard deviation of the residuals $(\mathrm{g} / \text { num. points })^{1 / 2}=419$ counts per pixel.

Two state-of-the-art techniques frequently used for the inverse Abel transform of flame images, BASEX [14, 19] and onion peeling with Tikhonov regularisation $[9,20]$, were compared to the FLiPPID method. For the Tikhonov regularisation, a zeroth-order smoothing matrix was used [21] while the regularisation parameter $\kappa$ was chosen such that significant smoothing of the reconstructed $R$ was achieved while avoiding large oscillations. L-curves [34] of numerous flame cross-sections for different colour channels were plotted to assure that $\kappa$ is within a sensible range (Fig. 10a). The results for BASEX without any smoothing/filtering $(q=1, \sigma=1)$ are also shown in Fig. 4 b.

For the smoothed BASEX $(q=2.2, \sigma=8)$ and onion-peeling technique, the $P(x, z)$ are hardly distinguishable from FLiPPID (Fig. 4a). However, both BASEX and onion peeling led to substantial noise in $R$, especially close to the flame centre (Fig. $4 \mathrm{~b}$ ). Both methods show a local maximum around $r=0$ which disappears just $0.15 \mathrm{~mm}$ away (Fig. 10b) and is thus attributed to noise. In contrast, Eq. 3 of FLiPPID is by definition a smooth function, including close to the axis of symmetry.

It is worth mentioning that fitting a function directly to $P(x)$ followed by the inverse transform Eq. 2 (very similar to the procedure by Deutsch [29]) was also attempted but proved to be impractical. Not even 10-parameter models for $P$ matched the performance of the FLiPPID method, either in terms of the quality of fit quantified by the objective function $g$ or in terms of the applicability at all HAB. In addition, fitting a function to $P$ led to similar issues as sometimes observed for the BASEX and onion-peeling techniques, including oscillations in $R$ and sometimes non-physical, negative values of $R$ near the central axis. The FLiPPID method a priori assumes a positive, smooth density and, therefore, does not suffer from such artifacts.

Once the emission source densities $R(r, z)$ are known for the three colour channels, the soot temperature $T$ can be computed. The relationship between the recorded colour ratio and the $T$ of the light-emitting incandescent material is given by [16]:

$$
\frac{R_{i}}{R_{j}}=\frac{\int_{0}^{\infty} \eta_{i}(\lambda) \frac{\epsilon(\lambda)}{\lambda^{5}}[\exp (h c / \lambda k T)-1]^{-1} \mathrm{~d} \lambda}{\int_{0}^{\infty} \eta_{j}(\lambda) \frac{\epsilon(\lambda)}{\lambda^{5}}[\exp (h c / \lambda k T)-1]^{-1} \mathrm{~d} \lambda},
$$

for the thermocouple and

$$
\frac{R_{i}}{R_{j}}=\frac{\int_{0}^{\infty} \eta_{i}(\lambda) \frac{\sigma_{\mathrm{ads}}(\lambda)}{\lambda^{3}}[\exp (h c / \lambda k T)-1]^{-1} \mathrm{~d} \lambda}{\int_{0}^{\infty} \eta_{j}(\lambda) \frac{\sigma_{\mathrm{ads}}(\lambda)}{\lambda^{3}}[\exp (h c / \lambda k T)-1]^{-1} \mathrm{~d} \lambda},
$$

for the soot particles. Here, $\lambda$ is the wavelength, $k$ and $h$ are the Boltzmann and Planck constants, $c$ is the speed of light, $\epsilon$ is the emissivity of the thermocouple and $\sigma_{\text {ads }}$ is an average absorption cross-section of the emitting soot particles. $R_{i}$ and $\eta_{i}(\lambda)$ are the reconstructed intensity and the camera response of the colour channel $i . \eta_{i}(\lambda)$ was obtained using the quantum efficiency of the camera and wavelength dependent filter transmission data provided by the respective manufacturers. For calibration, an R-type thermocouple was placed at different distances above a Bunsen burner and imaged. The detected colour ratios at different thermocouple temperatures (1570-1930 K) were used to calibrate the camera response [16]. The values reported by Ma and Long [17] were used for $\epsilon(\lambda)$ of the thermocouple. After calibration, the difference in the calculated and observed thermocouple temperatures for a given colour ratio were within $20 \mathrm{~K}$. Using the calibrated camera response in Eq. 7, the expected light colour of soot was calculated as a function of temperature. The results of the calculations were used to create a look-up table for the temperature as a function of the three different RGB ratios. Assigning a value of $\sigma_{\text {ads }}(\lambda)$ for soot is not straightforward as it is a function of the soot growth history and carbon/hydrogen ratio, and thus varies within the flame [35-38]. The optical properties of soot are beyond the scope of this work and the most commonly used dependence in the field $[13,16,36]$, $\lambda^{2} \sigma_{\text {ads }}(\lambda) \propto \lambda^{-1.38}$, was used.

Fig. 5a compares the calculated soot temperatures using FLiPPID, BASEX and onion-peeling for converting the recorded projected intensity profiles $P(x, z)$ to the emission intensity crosssection distributions $R(r, z)$. The shown temperatures were obtained using Eq. 7, with three different RGB ratios (R/G, R/B, $\mathrm{G} / \mathrm{B}$ ) and averaging the results as per [16]. The regularisation parameters for the BASEX and Tikhonov regularisation were the same as used in Fig. 4. All the other conditions (assumed $\sigma_{\text {ads }}$ for soot, raw flame image, the $T$ look-up table) were identical. The soot temperatures are in a similar range as the ones reported for similar co-flow diffusion flames using different fuels $[13,16]$. All three methods (FLiPPID, BASEX, onion-peeling with Tikhonov regularisation) gave qualitatively similar temperature distributions. However, BASEX and onion-peeling both gave noisy results close to the centre-line. Below $32 \mathrm{~mm} \mathrm{HAB}$, the centre-line temperatures were too noisy to obtain reliable 


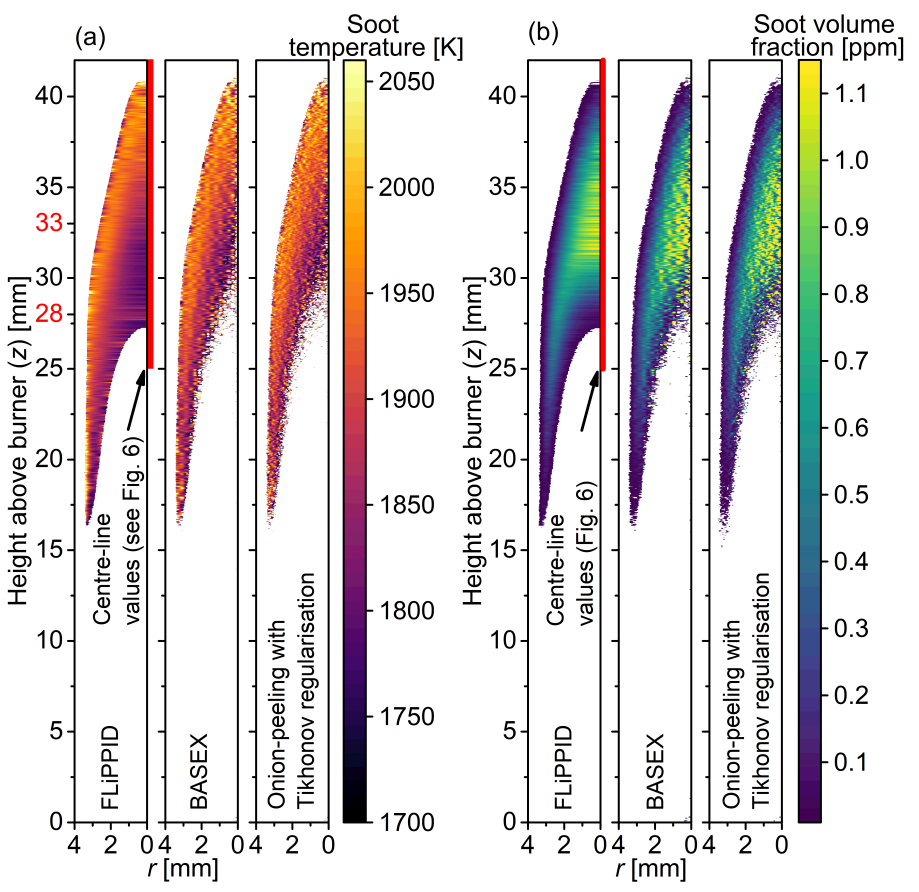

Fig. 5. Calculated (a) soot temperature $T$ and (b) volume fraction $f_{\mathrm{v}}$ using three different methods for performing the inverse Abel transform (see bottom labels). For $28 \mathrm{~mm}$ and 33 $\mathrm{mm} \mathrm{HAB}$, line plots of the intensity recorded with the green camera channel are plotted in Fig. 4. The distributions of $T$ and $f_{\mathrm{v}}$ over the highlighted centre-lines are shown in Fig. 6.

values. It should be noted that colour ratio pyrometry is particularly sensitive to noisy reconstructions because their ratios are required for the soot temperature. FLiPPID led to relatively smooth centre-line temperatures even down to $27 \mathrm{~mm} \mathrm{HAB}$ and due to the smooth reconstruction, does not suffer from noise amplification when taking colour ratios. The reduction in noise along the flame centre is further illustrated in Fig. 6a.

Once $T$ is known, the soot volume fraction $f_{\mathrm{v}}$ can be calculated $[16,17,39]$ :

$$
f_{\mathrm{v}}=-\frac{\lambda_{\text {eff }}}{K_{\text {ext }} L} \ln \left(1-\epsilon_{\mathrm{L}}\left(\lambda_{\text {eff }}\right) \frac{R_{\mathrm{i}}}{\tau S_{\mathrm{L}}}\right) \approx \frac{\lambda_{\text {eff }} \epsilon_{\mathrm{L}}}{K_{\text {ext }} L} \frac{R_{\mathrm{i}}}{\tau S_{\mathrm{L}}},
$$

(as it follows from Eqs. 1\&5 of Ref. [39]; the expansion of the natural logarithm in series is accurate for optically thin flames). Here, $\lambda_{\text {eff }}$ is the effective filter wavelength [17], $K_{\text {ext }}$ is the soot dimensionless extinction coefficient (value taken as 8.6 [16]), $L$ is the pixel dimension ( $1 \mathrm{~mm}$ per 34 pixels), and $\epsilon_{\mathrm{L}}\left(\lambda_{\text {eff }}\right)$ is the emissivity at $\lambda_{\text {eff }}$ of the calibration source (R-type thermocouple). The light emitted from a layer of soot particles, 1 pixel thick, which is recorded by colour channel $i$ using exposure time $\tau$ is $R_{i}$. For $R_{\mathrm{i}}$, the intensity of the green colour channel obtained by applying FLiPPID, BASEX, or onion-peeling was used. Images of the hot thermocouple provided the light intensity of the calibration source $\left(S_{\mathrm{L}}\right)$. An interpolation of $S_{\mathrm{L}}$ per exposure time as a function of the temperature was used in Eq. 8 at the respective soot temperature calculated previously (Fig. 5a).

The values of $f_{\mathrm{v}}$ calculated using the three Abel inversion methods are shown in Fig. 5b. The maximum value of $f_{\mathrm{v}}$ was around $1.1 \mathrm{ppm}$, which is in between values reported for coflow diffusion flames fed with methane $(0.08 \mathrm{ppm}[13,40])$ and ethylene (5-8 ppm $[16,36])$ and similar to a nitrogen diluted $n$-heptane flame operated at 2 bar $(0.8 \mathrm{ppm})$ [41]. Among the three methods for Abel inversion, the new FLiPPID method led to significantly smoother profiles. This is evident on the plot of the centre-line values of $f_{\mathrm{v}}$ against HAB (Fig. 6b). Note that for FLiPPID, single pixel centre-line values are shown in Fig. 6 while for BASEX and onion-peeling, averages of the central 11 pixels (corresponding to $0.32 \mathrm{~mm}$ ) were used instead to reduce the noise. Even then, the values obtained with BASEX and onion peeling were significantly noisier.
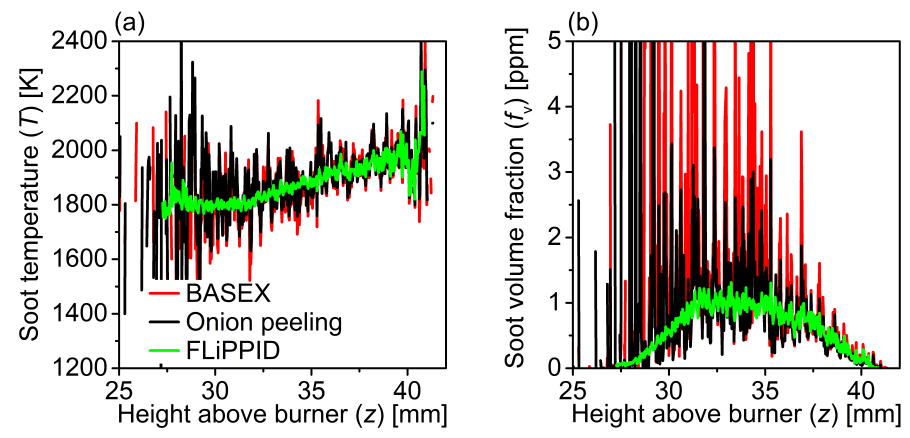

Fig. 6. Flame centre-line values for the soot temperature (a) and volume fractions (b) using three different methods for performing the inverse Abel transform.

The reproducibility of the three Abel inversion methods was analysed by processing 50 image frames taken during one experiment and comparing soot volume fractions (Fig. 7). Two different approaches were chosen to evaluate the images: (1) calculate the soot volume fraction for each individual frame and average the results and (2) average the 50 image frames and calculate the soot volume fraction using the averaged image. The shaded areas show the standard deviation $\left(\sigma_{f \mathrm{v}}\right)$ when method (1) was used and is a quantitative measure of the uncertainty bounds of the Abel inversion technique. Note that the average of method (1) is not shown as it would simply lie in the middle of $\sigma_{f_{\mathrm{v}}}$. At all $r$, onion peeling combined with Tikhonov regularisation led to significantly higher uncertainty bounds than FLiPPID. It can also be seen that for onion peeling, $\sigma_{f_{\mathrm{V}}}$ increases with decreasing $r$ and even reaches negative $f_{\mathrm{v}}$. In contrast, FLiPPID results in positive $f_{\mathrm{v}}$ at all $r$ with a maximum $\sigma_{f_{\mathrm{V}}}$ of $0.1 \mathrm{ppm}$. Processing an averaged image $\bar{P}_{\text {frames }}$ as per method (2) reduces the noise and its amplification towards the centre when using onion peeling (red lines, Fig. 7), as would be expected due to the less noisy input image. The absolute values of the $f_{\mathrm{v}}$ are now similar to the ones obtained by FLiPPID but overall still exhibit substantial noise. Thus, FLiPPID is capable of producing reliable soot volume fractions from a single image frame with tighter uncertainty limits than alternative methods produce from 50 averaged frames.

It is also worth noting that onion peeling appears to lead very often to several local extrema in the vicinity of $r=0$. Although such extrema in $f_{\mathrm{v}}$ were observed before for Abel inverted data [42], they are most likely an artifact caused by noise amplification towards the flame centre when conventional Abel inversion methods are used. The amplitudes of the maxima decrease when multiple frames are averaged to reduce the noise in the recorded data (compare the shaded area and lines in Fig. 7). In fact, the maximum at the central line for $z=28 \mathrm{~mm}$ completely 
disappeared for the averaged image (Fig. 7b). Furthermore, such sharp local extrema are not observed in experiments not relying on inverse Abel transforms [17, 32].
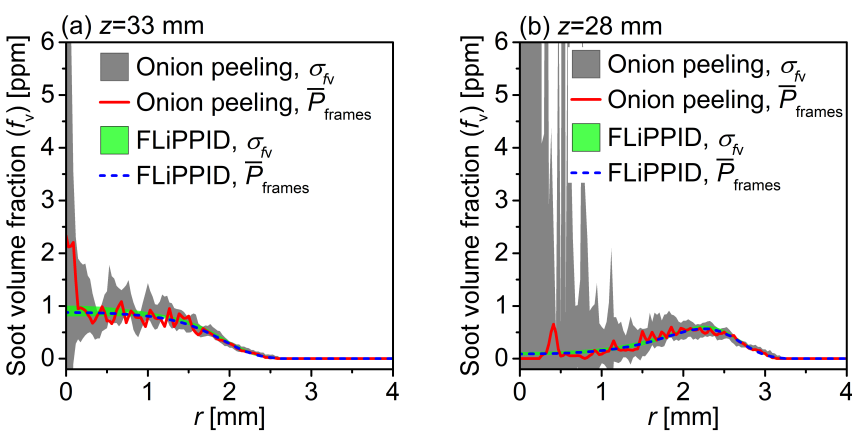

Fig. 7. Standard deviation of soot volume fractions when processing 50 individual image frames ( $\sigma_{f_{\mathrm{V}}}$; shaded area) and soot volume fractions obtained when processing the average of the same 50 frames ( $\bar{P}_{\text {frames }}$; lines). Results are presented for (a) 38 $\mathrm{mm}$ and (b) $28 \mathrm{~mm} \mathrm{HAB}$ using onion peeling combined with Tikhonov regularisation or FLiPPID.

Additional error analysis of FLiPPID is presented in Appendix C, where a 4th (dummy) parameter is introduced in $R$ and is varied so that the optimal residual sum-of-squares (after minimisation with respect to $a, b$ and $c$ ) remains below $3 \%$ of the intrinsic sum-of-squares. The hereby determined uncertainty bounds in the centre of the flame are $\pm 10 \%$, which is similar to the reproducibility of the recorded images (cf. the Appendix C).

\section{CONCLUSION}

In conclusion, the newly developed FLiPPID method enabled smooth reconstruction of flame cross-sections, even close to the flame centre-line, where other methods struggle. What distinguishes FLiPPID from the conventional Abel inversion techniques, such as BASEX, onion peeling combined with Tikhonov regularisation, or methods with polynomial, exp-polynomial or spline regression over $P$ (e.g., ref. [29]) are the imposed constraints on $R$ : the conditions for non-negativity and for simplicity. BASEX and onion peeling impose the less restrictive condition for smoothness only, and in result the noise damping is less efficient. Methods with direct regression of the projection $P$ often implicitly impose a simplicity condition on the test model for $P$, but not as strong as FLiPPID. For the flame images studied here, an exp-polynomial for $P$ requires 12-17 parameters to produce the same residual sum-of-squares as the best FLiPPID function, Eq. 3, produced with three. In addition, the $P$-regression methods cannot impose a non-negativity condition.

Thus, FLiPPID uses more of the available information for the specific system investigated, which improves the reconstruction significantly and avoids physically dubious or meaningless results (negative light intensities, multiple maxima). These advantages are at the expense of speed: with the current version of the Python code ([1] and [2] in Appendix B), the FLiPPID analysis of one image takes 39 min (not counting the integral tabulation; cf. Eq. 5). In comparison, BASEX and onion peeling take only seconds. In addition, the error analysis of FLiPPID is less straightforward as for other methods.

The obtained values for the soot temperature $T$ and volume fraction $f_{\mathrm{v}}$ were generally in a similar range as for commonly used Abel inversion methods (BASEX and onion peeling with Tikhonov regularisation). However, FLiPPID produced significantly less noisy reconstructed images compared to BASEX and onion-peeling, especially close to the flame centre. The predefined intensity distribution chosen here (Eq. 3) was optimised for co-flow diffusion flames and preliminary tests showed that Eq. 3 seems to be applicable to a wide range of such flames. It is expected that the FLiPPID methodology described here can also be applied to other experimental techniques employing the Abel transform (e.g., modulated absorption/emission [43, 44], laser extinction [42], or in-line holography $[18,45])$ simply by adjusting or extending Eq. 3.

\section{APPENDIX A: IMAGE PROCESSING}

The algorithm for calculating soot temperature and volume fraction profiles is schematically shown in Fig. 2. A brief description of each step is given below.

Import RGB flame image. The images were recorded in 12 bit raw format with a resolution of 1536x2048 pixel. The images were converted to TIF format using ImageJ and imported into Python. The RGB channels are obtained by debayering the raw image with the Python colour-demosaicing function.

Pre-process image. For performing the inverse Abel transform, the flame image has to be cropped such that the axis of symmetry is in the centre. The Python code automatically detects the flame edges by searching for the first and last column above a threshold and taking the middle of these as centre (Fig. 2 ). The procedure is repeated for a number of rows to obtain an averaged flame centre and to check that the flame is not tilted. The user-defined burner position (HAB0), crop width and crop height are used to cut the raw image from HAB0 to the crop height in the $z$-direction and to the crop width with the flame centre in the middle in $x$-direction (see Fig. 2).

Extract RGB channels. The cropped red, green, and blue colour channels of the image are extracted.

Performing inverse Abel transform. The inverse Abel transform of the image is calculated for each colour channel. The detailed flow chart of the FLiPPID method is shown in Fig. 3 and described below. Alternative methods such as the basis-set expansion (BASEX) $[18,19]$ and the onion-peeling method combined with a Tikhonov regularisation $[20,21]$ are described in detail in the literature.

Calculate colour ratios R/G, R/B, and B/G. The colour ratios of the reconstructed flame cross sections are calculated. If the intensity of one of the colour channels falls below a predefined threshold, the ratio at this pixel is set to 0 to prevent meaningless background colour ratios to be calculated.

Convert colour ratios to soot temperatures. The calculated colour ratios are converted to soot temperatures using a temperature look-up table. A description of how this table is obtained is given in [16].

Calculate soot volume fraction. Using Eq. 8, the previously obtained soot temperatures, the camera exposure time used while recording the flame image, and the camera calibration, the soot volume fractions are calculated.

Export soot temperature and volume fraction. The obtained soot temperature and volume fraction profiles are exported.

\section{APPENDIX B: FLIPPID ALGORITHM}

Import pre-processed RGB channels. Input to the FLiPPID method are matrices of the experimental 2D projections recorded 
by the camera and the predefined intensity distribution $R(r$; $a, b, c)$ (Eq. 3 in this study). The axis of symmetry of the experimental data has to be in the centre of the matrix.

Initial guess for $a, b, c$. The initial values of $a, b, c$ are selected. The values should be reasonable to assure fast convergence in the subsequent optimisation.

Select first colour channel $i$. The first RGB channel is selected.

Select first pixel row $z$. The first line of pixels is selected.

Calculate the forward Abel transform to obtain $P(x ; a, b, c)$. The line-of-sight projection of $R(r ; a, b, c)$ is calculated using the forward Abel transform (Eq. 4).

Compare the experimental data of colour channel $i$ at pixel row $z$ to $P(x ; a, b, c)$. The residual sum-of-squares objective function $g(z ; a, b, c, \ldots)$ for the calculated projection $P$ and the corresponding experimentally recorded $2 \mathrm{D}$ projection data is constructed.

Optimisation. The fitting parameters $a, b, c$ are determined by minimising the objective function $g$.

Last pixel row $z$. If the last pixel row $z$ of the input matrix was reached, the code checks if there are other colour channels to process. If the last $z$ was not reached the next pixel row is selected and the previously optimised $a, b, c$ are used as initial guess.

Last colour channel. If all three RGB channels were processed the program exits. Otherwise the next colour channel is selected.

Output optimised $a, b, c$ for each colour channel $i$ and pixel row $z$. Matrices of $a, b, c$ for the three colour channels as function of $z$ are exported. An example for the optimised parameters of the green colour channel is shown in Fig. 8.

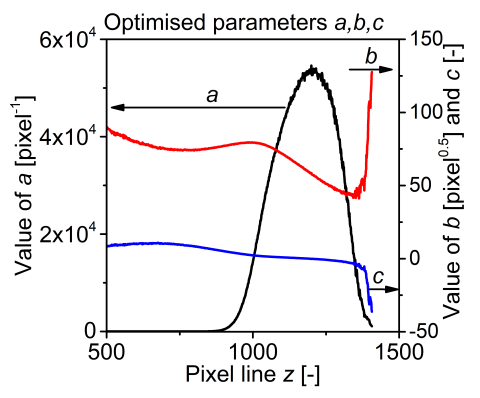

Fig. 8. Optimised fitting parameters $a, b, c$ for the green colour channel as function of pixel row $z$.

\section{Flame pyrometry and FLiPPID Python code}

1. J. A. H. Dreyer, R. I. Slavchov, and A. Menon, "FLiPPID flame pyrometry Python code" (Apollo University of Cambridge data repository, 2019), https://doi.org/10.17863/CAM.36025.

2. J. A. H. Dreyer, R. I. Slavchov, and A. Menon, "Flame pyrometry" (CoMo website, 2019), https://como.cheng.cam.ac.uk/index. php?Page=FlamePyrometry.

\section{APPENDIX C: FLIPPID ACCURACY}

Consider the following two 4-parametric functions:

$$
R(r)=\frac{a}{b \sqrt{\pi}} \exp \left[c\left(\frac{r}{b}\right)^{2}+d\left(\frac{r}{b}\right)^{4}-\left(\frac{r}{b}\right)^{6}\right],
$$
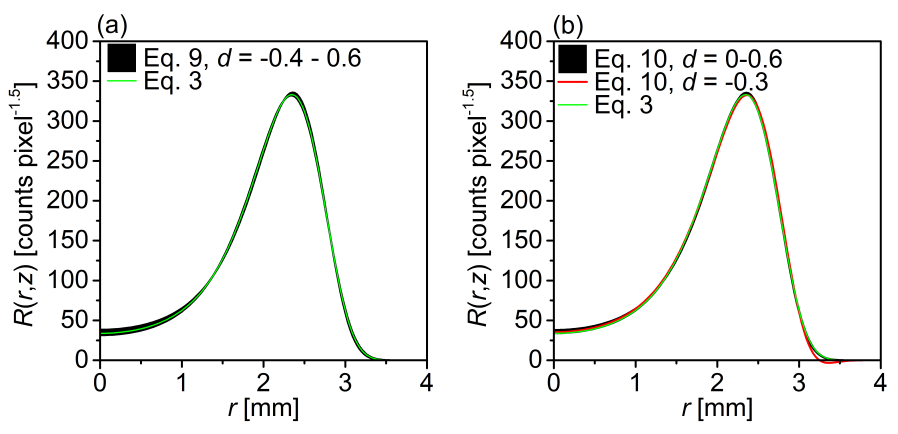

Fig. 9. A set of 4-parametric functions that produce residual sum-of-squares within $3 \%$ from that of the three-parametric Eq. 3. (a) Eq. 9; (b) Eq. 10. Green line is the 3-parameter function; the red line is the optimal Eq. 10 with the non-negativity constraint relaxed.

and

$$
R(r)=\frac{a}{b \sqrt{\pi}}\left(1+d\left(\frac{r}{b}\right)^{4}\right) \exp \left[c\left(\frac{r}{b}\right)^{2}-\left(\frac{r}{b}\right)^{6}\right] .
$$

These functions simplify to Eq. 3 when $d=0$. Since the optimised Eq. 3 is already very closed to the intrinsic sum-of-squares, the variation of the new parameter $d$ cannot decrease the sumof-squares significantly: the optimised standard deviation of the residuals of Eq. 3 is 427 counts per pixel (fluctuation 2.66\%), and the optimised Eqs. 9 and 10 both produce standard deviation of the residuals $0.2 \%$ lower than that of Eq. 3 for the given example $(z=28 \mathrm{~mm}$ HAB$)$. Accordingly, the parameter $d$ can be varied as a dummy parameter in a range of values; for each value of $d$, the other three parameters, $a, b$, and $c$, can be optimised with $g$ changing insignificantly. Thus, an infinite set of 4-parametric functions (note that $d$ was fixed) can be produced that show that same level of agreement with the data as the optimal Eq. 3 in terms of $g$. The subset of functions for which $g$ is within $3 \%$ of the value of $g$ for the optimised Eq. 3 is plotted in Fig. 9, as a shaded area. This plot is informative in 3 respects. First, the spread of the sub-set is a measure of the uncertainty bounds of FLiPPID. According to both Fig. 9a and b, the sum-of-squares varies by less than $3 \%$ for an infinite set of models for $R$ that differ at most by $\pm 10.2 \%$ (the maximum difference between Eq. 3 and Eq. 10, which is in the centre of the flame) or within $+12 \%$ for Eq. 9 . Second, the analysis demonstrates the effect of relaxing the simplicity constraint: adding one parameter to the model function improves the fit insignificantly, but adds to the uncertainty of the result. The simplest function able to explain the observed data is the best one can hope for; any additional features (parameters) should be considered dubious. Third, the second function, Eq. 10 , allows also the non-negativity constraint to be relaxed. The global minimum of the residual sum-of-squares of this function and the data is at $d=-0.3$. However, this value leads to unphysical negative light intensity at the edge of the flame (Fig. 9b, red line). We should note that it is often the case with our data that if the non-negativity condition is relaxed, the optimised $R$ shows a negative region (the optimal function is rarely positive). We believe that this is not a coincidence and is related to the optically thin flame approximation. The recorded projection is likely affected by self-absorption, which should cause a drop of $P$ near the flame centre. Our preliminary analysis suggests that 
this should result in a tendency for a positive average residual near the centre when non-negativity is imposed, and negative values of $R$ near the wings when this constraint is relaxed (we indeed observe both effects). In a future study, we will attempt to correct for the self-absorption.

\section{APPENDIX D: SUPPORTING FIGURES}
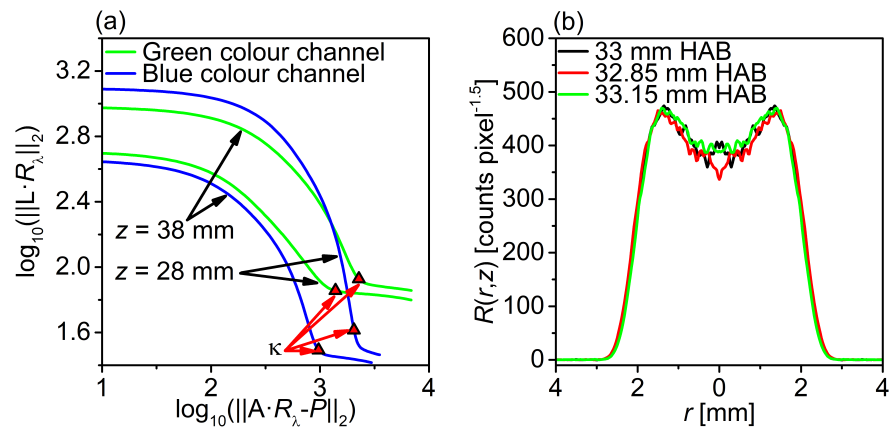

Fig. 10. (a) Selected L-curves for the onion peeling method with Tikhonov regularisation showing that the chosen values of $\kappa$ are close to the L corners. (b) $R$ close to $33 \mathrm{~mm}$ HAB obtained using onion peeling with Tikhonov regularisation indicating that the extrema around $r=0$ are most likely produced by noise.

\section{FUNDING.}

This project is funded by the National Research Foundation, Prime Minister's Office, Singapore under its CREATE programme.

\section{REFERENCES}

1. A. D. Abid, J. Camacho, D. A. Sheen, and H. Wang, "Quantitative measurement of soot particle size distribution in premixed flames - The burner-stabilized stagnation flame approach," Combust. Flame 156, 1862-1870 (2009).

2. M. Conturso, M. Sirignano, and A. D'Anna, "Effect of $\mathrm{C}_{9} \mathrm{H}_{12}$ alkylbenzenes on particle formation in diffusion flames: An experimental study," Fuel 191, 204-211 (2017).

3. T. Zhang, L. Zhao, and M. J. Thomson, "Effects of n-propylbenzene addition to $n$-dodecane on soot formation and aggregate structure in a laminar coflow diffusion flame," Proc. Combust. Inst. 36, 1339-1347 (2017).

4. Y. R. Tan, M. L. Botero, Y. Sheng, J. A. H. Dreyer, R. Xu, W. Yang, and M. Kraft, "Sooting characteristics of polyoxymethylene dimethyl ether blends with diesel in a diffusion flame," Fuel. 224, 499-506 (2018).

5. E. D. Tolmachoff, A. D. Abid, D. J. Phares, C. S. Campbell, and H. Wang, "Synthesis of nano-phase $\mathrm{TiO}_{2}$ crystalline films over premixed stagnation flames," Proc. Combust. Inst. 32, 1839-1845 (2009).

6. G. De Falco, A. Porta, A. M. Petrone, P. Del Gaudio, A. El Hassanin, M. Commodo, P. Minutolo, A. Squillace, and A. D'Anna, "Antimicrobial activity of flame-synthesized nano- $\mathrm{TiO}_{2}$ coatings," Environ. Sci.: Nano 4, 1095-1107 (2017).

7. N. K. Memon, F. Xu, G. Sun, S. J. B. Dunham, B. H. Kear, and S. D. Tse, "Flame synthesis of carbon nanotubes and few-layer graphene on metal-oxide spinel powders," Carbon 63, 478-486 (2013).

8. M. Y. Manuputty, J. A. H. Dreyer, Y. Sheng, E. J. Bringley, M. Botero, J. Akroyd, and M. Kraft, "Polymorphism of nanocrystalline $\mathrm{TiO}_{2}$ prepared in a stagnation flame: formation of the $\mathrm{TiO}_{2}$-Il phase," Chem. Sci. 10, 1342-1350 (2019).
9. N. J. Kempema and M. B. Long, "Effect of soot self-absorption on color-ratio pyrometry in laminar coflow diffusion flames," Opt. Lett. 43, 1103-1106 (2018).

10. A. Naseri, A. Veshkini, and M. J. Thomson, "Detailed modeling of $\mathrm{CO}_{2}$ addition effects on the evolution of soot particle size distribution functions in premixed laminar ethylene flames," Combust. Flame 183, 75-87 (2017).

11. M. L. Botero, N. Eaves, J. A. Dreyer, Y. Sheng, J. Akroyd, W. Yang, and M. Kraft, "Experimental and numerical study of the evolution of soot primary particles in a diffusion flame," Proc. Combust. Inst. 37, 2047-2055 (2019).

12. M. L. Botero, Y. Sheng, J. Akroyd, J. Martin, J. A. Dreyer, W. Yang, and M. Kraft, "Internal structure of soot particles in a diffusion flame," Carbon. 141, 635-642 (2019).

13. D. D. Das, W. J. Cannella, C. S. McEnally, C. J. Mueller, and L. D. Pfefferle, "Two-dimensional soot volume fraction measurements in flames doped with large hydrocarbons," Proc. Combust. Inst. 36, 871879 (2017).

14. D. D. Das, P. C. St. John, C. S. McEnally, S. Kim, and L. D. Pfefferle, "Measuring and predicting sooting tendencies of oxygenates, alkanes, alkenes, cycloalkanes, and aromatics on a unified scale," Combust. Flame 190, 349-364 (2018).

15. H. Guo, J. A. Castillo, and P. B. Sunderland, "Digital camera measurements of soot temperature and soot volume fraction in axisymmetric flames," Appl. Opt. 52, 8040-8047 (2013).

16. P. B. Kuhn, B. Ma, B. C. Connelly, M. D. Smooke, and M. B. Long, "Soot and thin-filament pyrometry using a color digital camera," Proc. Combust. Inst. 33, 743-750 (2011).

17. B. Ma and M. B. Long, "Absolute light calibration using S-type thermocouples," Proc. Combust. Inst. 34, 3531-3539 (2013).

18. M. Apostolopoulos, M. Taroudakis, and D. Papazoglou, "Application of inverse Abel techniques in in-line holographic microscopy," Opt. Commun. 296, 25-34 (2013).

19. V. Dribinski, A. Ossadtchi, V. A. Mandelshtam, and H. Reisler, "Reconstruction of Abel-transformable images: The Gaussian basis-set expansion Abel transform method," Rev. Sci. Instrum. 73, 2634-2642 (2002).

20. C. J. Dasch, "One-dimensional tomography: A comparison of Abel, onion-peeling, and filtered backprojection methods," Appl. Opt. 31, 1146-1152 (1992).

21. K. J. Daun, K. A. Thomson, F. Liu, and G. J. Smallwood, "Deconvolution of axisymmetric flame properties using Tikhonov regularization," Appl. Opt. 45, 4638-4646 (2006).

22. P. S. Kolhe and A. K. Agrawal, "Abel inversion of deflectometric data: comparison of accuracy and noise propagation of existing techniques," Appl. Opt. 48, 3894-3902 (2009).

23. M. J. Buie, J. T. P. Pender, J. P. Holloway, T. Vincent, P. L. G. Ventzek, and M. L. Brake, "Abel's inversion applied to experimental spectroscopic data with off axis peaks," J. Quant. Spectrosc. Radiat. Transf. 55, 231-243 (1996).

24. L. M. Smith, D. R. Keefer, and S. I. Sudharsanan, "Abel inversion using transform techniques," J. Quant. Spectrosc. Radiat. Transf. 39, 367-373 (1988).

25. S. Ma, H. Gao, and L. Wu, "Modified fourier-hankel method based on analysis of errors in abel inversion using fourier transform techniques," Appl. Opt. 47, 1350-1357 (2008).

26. P. A. Vicharelli and W. A. Lapatovich, "Iterative method for computing the inverse abel transform," Appl. Phys. Lett. 50, 557-559 (1987).

27. M. P. Freeman and S. Katz, "Determination of the radial distribution of brightness in a cylindrical luminous medium with self-absorption," J. Opt. Soc. Am. 50, 826-830 (1960).

28. C. D. Maldonado, A. P. Caron, and H. N. Olsen, "New method for obtaining emission coefficients from emitted spectral intensities. part I-circularly symmetric light sources," J. Opt. Soc. Am. 55, 1247-1254 (1965).

29. M. Deutsch, "Abel inversion with a simple analytic representation for experimental data," Appl. Phys. Lett. 42, 237-239 (1983).

30. C. S. McEnally and L. D. Pfefferle, "Improved sooting tendency mea- 
surements for aromatic hydrocarbons and their implications for naphthalene formation pathways," Combust. Flame 148, 210-222 (2007).

31. C. S. McEnally and L. D. Pfefferle, "Sooting tendencies of oxygenated hydrocarbons in laboratory-scale flames," Environ. Sci. Technol. 45, 2498-2503 (2011).

32. C. S. McEnally, A. M. Schaffer, M. B. Long, L. D. Pfefferle, M. D. Smooke, M. B. Colket, and R. J. Hall, "Computational and experimental study of soot formation in a coflow, laminar ethylene diffusion flame," Proc. Combust. Inst. 27, 1497-1505 (1998).

33. K. T. Walsh, J. Fielding, and M. B. Long, "Effect of light-collection geometry on reconstruction errors in abel inversions," Opt. Lett. 25, 457-459 (2000).

34. P. C. Hansen and D. P. O'Leary, "The use of the I-curve in the regularization of discrete ill-posed problems," SIAM J. Sci. Comput. 14, 1487-1503 (1993).

35. N. J. Kempema, B. Ma, and M. B. Long, "Investigation of in-flame soot optical properties in laminar coflow diffusion flames using thermophoretic particle sampling and spectral light extinction," Appl. Phys. B 122, 232 (2016).

36. S. De luliis, M. Barbini, S. Benecchi, F. Cignoli, and G. Zizak, "Determination of the soot volume fraction in an ethylene diffusion flame by multiwavelength analysis of soot radiation," Combust. Flame 115, 253-261 (1998).

37. F. Liu, K. A. Thomson, and G. J. Smallwood, "Soot temperature and volume fraction retrieval from spectrally resolved flame emission measurement in laminar axisymmetric coflow diffusion flames: Effect of self-absorption," Combust. Flame 160, 1693-1705 (2013).

38. B. Ma and M. B. Long, "Combined soot optical characterization using 2-D multi-angle light scattering and spectrally resolved line-of-sight attenuation and its implication on soot color-ratio pyrometry," Appl. Phys. B 117, 287-303 (2014).

39. F. Cignoli, S. De luliis, V. Manta, and G. Zizak, "Two-dimensional twowavelength emission technique for soot diagnostics," Appl. Opt. 40, 5370-5378 (2001).

40. D. D. Das, C. S. McEnally, T. A. Kwan, J. B. Zimmerman, W. J. Cannella, C. J. Mueller, and L. D. Pfefferle, "Sooting tendencies of diesel fuels, jet fuels, and their surrogates in diffusion flames," Fuel. 197, 445-458 (2017).

41. A. E. Karataş, G. Intasopa, and Ö. L. Gülder, "Sooting behaviour of $n$-heptane laminar diffusion flames at high pressures," Combust. Flame 160, 1650-1656 (2013)

42. M. Kashif, J. Bonnety, P. Guibert, C. Morin, and G. Legros, "Soot volume fraction fields in unsteady axis-symmetric flames by continuous laser extinction technique." Opt. Express 20, 28742-28751 (2012).

43. B. Franzelli, M. Roussillo, P. Scouflaire, J. Bonnety, R. Jalain, T. Dormieux, S. Candel, and G. Legros, "Multi-diagnostic soot measurements in a laminar diffusion flame to assess the ISF database consistency," Proc. Combust. Inst. 37, 1355-1363 (2019).

44. G. Legros, Q. Wang, J. Bonnety, M. Kashif, C. Morin, J.-L. Consalvi, and F. Liu, "Simultaneous soot temperature and volume fraction measurements in axis-symmetric flames by a two-dimensional modulated absorption/emission technique," Combust. Flame 162, 2705-2719 (2015).

45. D. G. Papazoglou and S. Tzortzakis, "In-line holography for the characterization of ultrafast laser filamentation in transparent media," Appl. Phys. Lett. 93, 041120 (2008). 